\title{
EL NIÑO 1997-98 EN LOS MEDIOS DE COMUNICACIÓN: REALIDADES, DEFORMACIONES Y EXAGERACIONES DE UN EVENTO CLIMÁTICO
}

\author{
Óscar Marcos Valiente*
}

\section{RESUMEN}

El fenómeno El Niño de 1997-98 ha sobrepasado su dimensión climática y ha adquirido una repercusión mediática sin precedentes, tomando temporalmente el relevo a otras preocupaciones medioambientales como el cambio climático o el agujero de la capa de ozono. Este artículo revisa el tratamiento informativo del evento, y ofrece una recopilación de curiosidades y equívocos relacionados con El Niño aparecidos en medios de comunicación.

Palabras clave: El Niño, ENSO, Internet, prensa, televisión.

\section{ABSTRACT}

The 1997-98 El Niño event has surpassed its climatic dimension and has acquired an unprecedented attention in the media, taking a temporary relay to other environmental matters such as climate change or the ozone layer hole. This article takes a view to the informative treatment of the event, offering also a summary of rarities and misunderstandings related to El Niño appeared in the media.

Keywords: El Niño, ENSO, Internet, press, television.

\section{Introducción}

Hemos asistido durante los últimos años a un creciente interés en los medios de comunicación por cualquier aspecto relacionado con el medio ambiente, y la climatología no es ajena a este proceso. Desde la información meteorológica, la más cercana a la pobla-

\footnotetext{
* Dept. Geografia Física i A.G.R. Universitat de Barcelona.
} 
ción por su constante repercusión sobre la actividad humana (trabajo, transporte aéreo y por carretera, tiempo de ocio, etc.), hasta diversas preocupaciones de índole climatológica, como el cambio climático o el agujero en la capa de ozono, el número de noticias dedicadas al tiempo y el clima que encontramos en prensa, radio y televisión se han multiplicado, facilitando que la sociedad haya asimilado conceptos e ideas propias de nuestra disciplina.

Durante los años 1997 y 1998 hemos sido testigos de la socialización de una nueva estrella del firmamento climatológico: El Niño. Este fenómeno, conocido ahora por el acrónimo ENSO (El Niño-Oscilación del Sur) por su doble componente oceánico y atmosférico, fue un desconocido entre la comunidad científica hasta la década de los 70 (Marcos, 1999), adquirió su mayoría de edad entre los estudiosos en los años 80 , y ha sido conocido entre toda la población a finales de los 90.

El geógrafo chileno César Caviedes llevó a cabo en 1984 (Caviedes, 1984) una recopilación de la atención suscitada por el fenómeno El Niño a través del número de artículos publicados en las principales revistas anglosajonas de las disciplinas de meteorología (Monthly Weather Review, Journal of Atmospheric Sciences, Journal of Applied Meteorology, Tellus y Weatherwise), oceanografía (Journal of Physical Oceanography, Oceanus, Journal of Marine Research y Deep Sea Research), geografía (Geographical Review, GeoJournal, Geoforum, Annal of the A.A.G., Geographic Journal, Geography, Professional Geographer y Progress in Physical Geography), medio ambiente (Discover, International Wildlife, Ecology, American Naturalist y Wildlife), y ciencias en general (Science, Science News, Nature, American Scientist, Scientific American y Smithsonian), y en revistas de información general publicadas en Estados Unidos (Newsweek y Time).

El resultado fue revelador. En el decenio comprendido entre 1972 y 1981, el número total de artículos publicados fue de 50, mientras que sólo en dos años (coincidiendo con El Niño de 1982-83), se contabilizan 45 referencias más. Especialmente significativo es el dato referido a las revistas de información general, puesto que El Niño no recibió atención alguna entre 1972 y 1981, mientras que el evento de 1982-83 mereció ocho artículos (seis en Newsweek y dos en la revista Time). La conclusión que podemos extraer es que El Niño ya comenzaba a ser conocido por la comunidad científica anglosajona en la década de los setenta, mientras que el público estadounidense tuvo las primeras noticias sobre la existencia del fenómeno a raíz del episodio de 1982-83.

En Europa, las referencias al fenómeno fueron cayendo a cuentagotas. La comunidad científica prestaba poco interés a un elemento del clima con incidencia escasa o nula en nuestro espacio geográfico, y los medios de comunicación dedicaron su espacio y su tiempo desde finales de los años 80 a mostrar las nefastas consecuencias del cambio climático o del debilitamiento de la capa de ozono en latitudes polares.

El mismo proceso de socialización del fenómeno El Niño experimentado en Estados Unidos tras el evento de 1982-83, se repitió en Europa con el episodio de 1997-98. Ambos eventos ENSO tuvieron una gran intensidad y provocaron anomalías climáticas (las denominadas teleconexiones) en diversos lugares del planeta, pero su tratamiento informativo ha sido distinto. En 1982 y 1983, los medios de comunicación se limitaron a presentar a la nueva estrella, sin poder profundizar en sus características, puesto que ni la sociedad estaba preparada para asimilar la complejidad del fenómeno, ni la comunidad científica disponía de suficiente información. En 1997 y 1998, las cadenas de radio y televisión, los periódicos y las revistas de información general, nos han bombardeado con noticias sobre El Niño, dado el extenso conocimiento científico generado en años anteriores. 
En Estados Unidos, la información relativa al fenómeno ENSO ha podido ser asimilada con mayor facilidad, al existir una fuerte relación entre el evento y las anomalías climáticas sobre Norteamérica, y haber tenido noticias de los sucesivos El Niño acaecidos en los 80 y los 90 . Al mismo tiempo, El Niño ya no es sólo objeto de tratamiento informativo, sino también humorístico y publicitario. De hecho, en el lenguaje coloquial estadounidense el vocablo El Niño se asocia a cualquier tipo de desastre, sea del ámbito que sea, y se aplica a personas que, a una corta edad, muestran habilidades inesperadas. Tal es el caso del golfista español Sergio García, quien recibió mientras jugaba torneos como aficionado en Estados Unidos el apodo de El Niño no sólo por su juventud (alcanzó la mayoría de edad en 1998), sino por ser considerado por los expertos como un fenómeno de la naturaleza, un vendaval de pericia y habilidad.

En Europa, donde no existía la experiencia informativa estadounidense, ha habido una avalancha de informaciones ligada al evento de 1997-98, en algunos casos inconexa y desordenada, por lo que la sociedad ha recibido una imagen algo distorsionada del fenómeno, confundiéndolo con otros conceptos climátológicos como el cambio climático. En cualquier caso, tanto en Estados Unidos como en Europa, El Niño ha sido objeto de exageraciones y deformaciones, provocando entre la población una imagen del mismo un tanto demoníaca.

En los siguientes apartados se presenta una recopilación orientativa de la información generada en torno al fenómeno ENSO en diversos ámbitos de la comunicación. No se trata de presentar un compendio exhaustivo de las noticias y artículos científicos aparecidos en 1997 y 1998, puesto que pueden contarse por miles en los medios de todo el mundo y por cientos en los españoles, sino de reflejar el súbito interés por un fenómeno que viene produciéndose desde hace miles de años.

\section{El niño en los medios escritos españoles}

Todos los medios de comunicación han hecho referencia a El Niño en repetidas ocasiones entre junio de 1997 y abril de 1998, período durante el cual se registró la fase de máxima incidencia del fenómeno. Sin embargo, en la hemeroteca española ya se encuentran referencias puntuales al fenómeno de una forma genérica meses antes:

- en El País Semanal («Hablemos del tiempo», 31 de marzo de 1996, nº 1018, pp. 5664), se mencionan tanto El Niño como La Niña, en relación con la caoticidad del sistema climático;

- en el diario El País («El clima de una zona del Pacífico permite prever las sequías del sureste español», 16 de enero de 1997), se hacía referencia a un estudio de dos ecólogos de la Universitat de Barcelona (Francisco Comín y Xavier Rodó) que defienden la existencia de una teleconexión entre El Niño y las sequías del ámbito peninsular más árido.

Si con estas noticias la prensa puso en antecedentes a los lectores sobre la existencia de El Niño, las informaciones sobre el evento de 1997-98 se retrasaron hasta el 6 de junio de 1997, fecha en la que La Vanguardia publicaba el artículo «Vuelve El Niño», advirtiendo que los satélites de medición de las variaciones en el nivel del mar del Pacífico ya anunciaban el inicio del fenómeno para el otoño de ese año. 
Durante los meses posteriores la sucesión de noticias fue interminable en todos los periódicos (sólo se muestran algunos ejemplos), presentando el fenómeno a través de sus efectos o la previsión de consecuencias en diversos lugares del planeta:

- 27 de julio de 1997: La Vanguardia, «El Niño se enfurece»;

- 7 de agosto de 1997: El País, «Filipinas se prepara ante la sequía con que amenaza El Niño»;

- 24 de agosto de 1997: $A B C$, «El fenómeno meteorológico 'El Niño' amenaza las costas de Califormia con graves inundaciones»;

- 28 de agosto de 1997: La Vanguardia, «Científicos advierten del efecto devastador de 'El Niño' en 1998»;

- 5 de septiembre de 1997: El País, «Perú estima que El Niño dejará pérdidas de 1.200 millones de dólares»;

- 18 de septiembre de 1997: El País, «El Niño causa pérdidas elevadas en El Salvador y Centroamérica»;

- 22 de septiembre de 1997: El Periódico de Catalunya, «El Niño da sus primeros latigazos»;

- 9 de octubre de 1997: El País, «El Niño costará 13.000 millones de dólares en todo el mundo»;

- 24 de noviembre de 1997: Avui, «La UB estudia el impacto del Niño sobre las lluvias de Catalunya»;

- 14 de febrero de 1998: El Periódico de Catalunya, «El Niño causará estragos hasta el verano»;

- 24 de febrero de 1998: Avui, «Una serie de tornados provoca al menos 43 muertos en Florida»;

- 2 de marzo de 1998): La Vanguardia, «Los científicos asocian el 'Niño' a una corriente de profundidad media»;

- 16 de marzo de 1998: El País, «El fenómeno de 'el Niño’ causa el desbordamiento del río Rimac cerca de Lima»;

Los suplementos dominicales de estos mismos periódicos dedicaron algunos de sus reportajes a explicar, de forma más didáctica que en los artículos de la prensa diaria, en qué consiste El Niño y cuáles son sus efectos. Algunos ejemplos se pueden encontrar en el Magazine de La Vanguardia («Atacama, la guarida del Niño», 1 de marzo de 1998, pp. 58-64), la Revista de La Vanguardia («El 'Niño' del cambio climático», 5 de octubre de 1997) o El País Semanal («El Niño más terrible», 21 de diciembre de 1997, nº 1108, pp. 86-96).

Entre las pocas excepciones al tratamiento informativo hasta ahora presentado, se encuentra un artículo en tono de sutil jocosidad de Horacio Sáenz Guerrero en el Magazine de La Vanguardia («Del 'Nino' al 'Niño'», 30 de noviembre de 1997, p. 98). Tras presentar algunos de los componentes del fenómeno, el autor se congratula de que un vocablo con la letra ñ haya adquirido una dimensión mundial:

«El 'Niño', pues, ya es el 'Niño', aunque de Jesús, nada. Tiene todo el furor de la madre naturaleza cuando le da por convertirse en madrastra».

En el resto de Europa y en todo el mundo, periódicos y revistas de información general dedicaron al fenómeno una atención similar e incluso superior. Para no extender los ejemplos ad infinitum, tan sólo destacaré que El Niño fue objeto de extensos reportajes en Time 
(1997, 1998), Veja (1997a, b, c), San Francisco Examiner (1997), U.S. News (1997), Der Spiegel (1997), Ercilla (1997) y Tyden (1998).

Otro tipo de publicaciones en las que El Niño ha estado muy presente han sido las revistas científicas. El Niño de 1982-83 ya había sido objeto de un extenso reportaje de National Geographic en febrero de 1984 («El Niño’s ill wind, pp. 144-183), mientras que el evento de 1997-98 protagonizó la portada del número de marzo de 1999 («El Niño / La Niña. El ciclo vicioso de la naturaleza», pp. 74-97). Ambos artículos presentan los efectos sobre el clima, la biología y la economía en todo el mundo, exponiendo el grado de conocimiento científico del fenómeno. Una comparación de los reportajes permite observar el grado de evolución de la ciencia entorno a El Niño en los últimos quince años.

El resto de revistas de divulgación científica publicadas en España también ofrecieron artículos de muy diversa índole dedicados a El Niño:

- En Ciencia y Vida (mayo de 1998, n 3, pp. 61-68), bajo el título «El Niño. La corriente loca que altera el clima», se ofrecen algunas de las posibles causas de la génesis del fenómeno, defendiendo la tesis de que la salinidad controla el ciclo oceánico en el Pacífico;

- En Mundo Científico (febrero de 1998, no 187, pp. 59-65): «Del fuego al agua: El Niño y La Niña». El artículo es presentado en la portada con el confuso titular de «Cambio climático. El Niño y sus efectos devastadores», aunque en el texto interior no se trata la relación entre ambos conceptos climáticos, sino que expone con claridad en qué consiste el fenómeno, cuáles son su efectos, la dinámica oceánico-atmosférica del Pacífico y el desarrollo del episodio de 1997-98. Ejemplos como éste abundado en demasía durante el evento de 1997-98, afirmando con excesiva ligereza que el cambio climático está aumentando la frecuencia e intensidad de El Niño

- En Newton (mayo de 1998, n 1, pp. 98-106), «El Niño y sus caprichos» presenta las características del fenómeno y especula sobre su incidencia en Europa.

- En Geo (noviembre de 1997, n 30, pp. 122-127), El Niño es presentado de forma secundaria en relación con el cambio climático en el artículo «Pronóstico: se espera un ascenso de las temperaturas».

Finalmente, merecen ser destacadas las referencias a El Niño en obras de referencia en formato multimedia, las denominadas enciclopedias digitales. Si hasta ahora resultaba difícil encontrar información sobre este evento climático en cualquier enciclopedia, las nuevas ediciones (tanto en formato papel como en CD-ROM o DVD-ROM) surgidas tras el fenómeno de 1997-98 incorporan información creciente. Dos son los ejemplos escogidos para ilustrar esta evolución, la enciclopedia Encarta 97 (Microsoft, 1997) y la enciclopedia multimedia sobre temas científicos La Aventura de la Ciencia (La Vanguardia, 1999). En el primer caso, se dedica a El Niño un artículo monográfico firmado por George Philander, profesor de la Universidad de Princeton, en el que se exponen las principales características del fenómeno (la información contenida es previa al inicio del severo evento de 1997-98). La segunda enciclopedia multimedia ofrece un breve vídeo en el que se explica qué es El Niño y cuáles son sus principales efectos, sin hacer mención al fenómeno de 1997-98 (pese a haber sido publicado en 1999, la información ofrecida en este CD-ROM también es previa al último evento).

En el conjunto de artículos y noticias presentados, El Niño ha sido objeto de un tratamiento informativo adecuado, aunque con ciertos tintes catastrofistas. El principal error aparecido en los medios ha sido el de relacionar el fenómeno con el cambio climático, lo 
que ha creado entre la opinión pública la impresión de que El Niño es producto de la actividad humana. Nada más lejos de la realidad, cuando existen registros del fenómeno que aseguran su existencia durante los últimos 10.000 años. En el próximo apartado, se presentan las mayores barbaridades, curiosidades y pinceladas de humor aparecidas en medios de comunicación estadounidenses durante El Niño 1997-98, que demuestran cómo en aquél país el fenómeno del Pacífico ha superado la fase de conocimiento de su existencia, y es ya objeto de un uso mercantilista y humorístico plagado de exageraciones e inexactitudes.

\section{El niño en los medios de EE.UU.: Un vocablo cotidiano}

Lo que más sorprende en el análisis sociológico del fenómeno El Niño en Estados Unidos, es el alto nivel de integración del vocablo en el lenguaje cotidiano, de tal forma que decir "El Niño" es sinónimo de caos, y cuando no se encuentra explicación a algún suceso, la solución más común es echarle la culpa a El Niño. Una muestra de la incorporación del fenómeno a la cultura popular estadounidense en su inclusión en el diálogo de una serie de televisión: en NYPD Blue (emitida en España por la cadena Telecinco con el título Policías de Nueva York), el detective Bobby Simone (encarnado por el actor Jimmy Smits), al conducir con su compañero Andy Sipowicz (Dennis Franz) por un paisaje anómalamente sin nieve en la Nueva York rural, pregunta " $¿ Y$ esta temperatura? A que se debe, ¿al cambio climático? ¿a El Niño?” (USA TODAY, 1996).

A esta incorporación del fenómeno al lenguaje cotidiano contribuyeron los medios de comunicación estadounidenses en todas sus vertientes: artículos escritos, reportajes en los

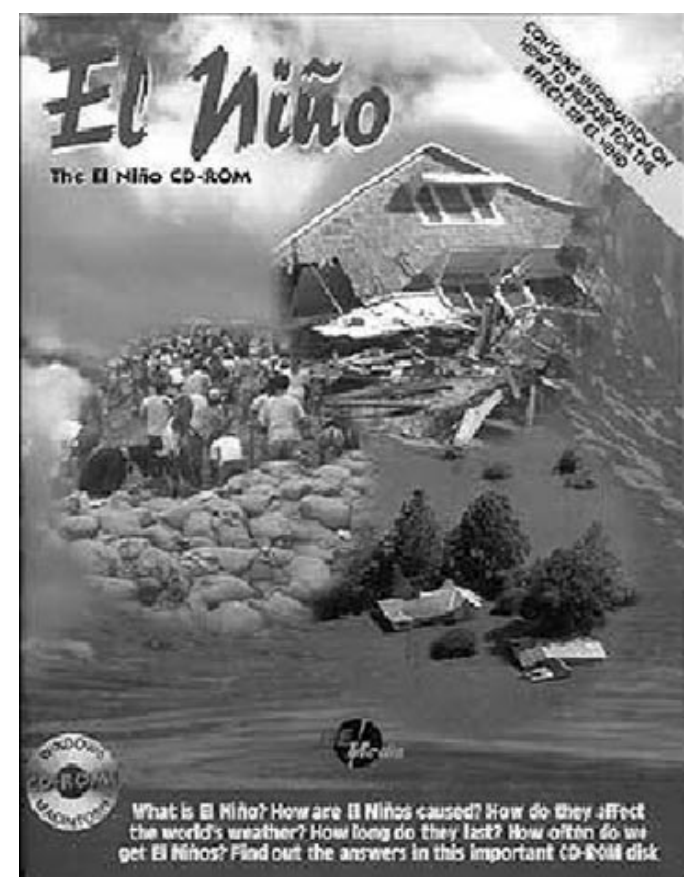

FIGURA 1. Portada del CD-ROM divulgativo sobre El Niño, publicado en Estados Unidos en febrero de 1998 (REMEDIA, 1998). 


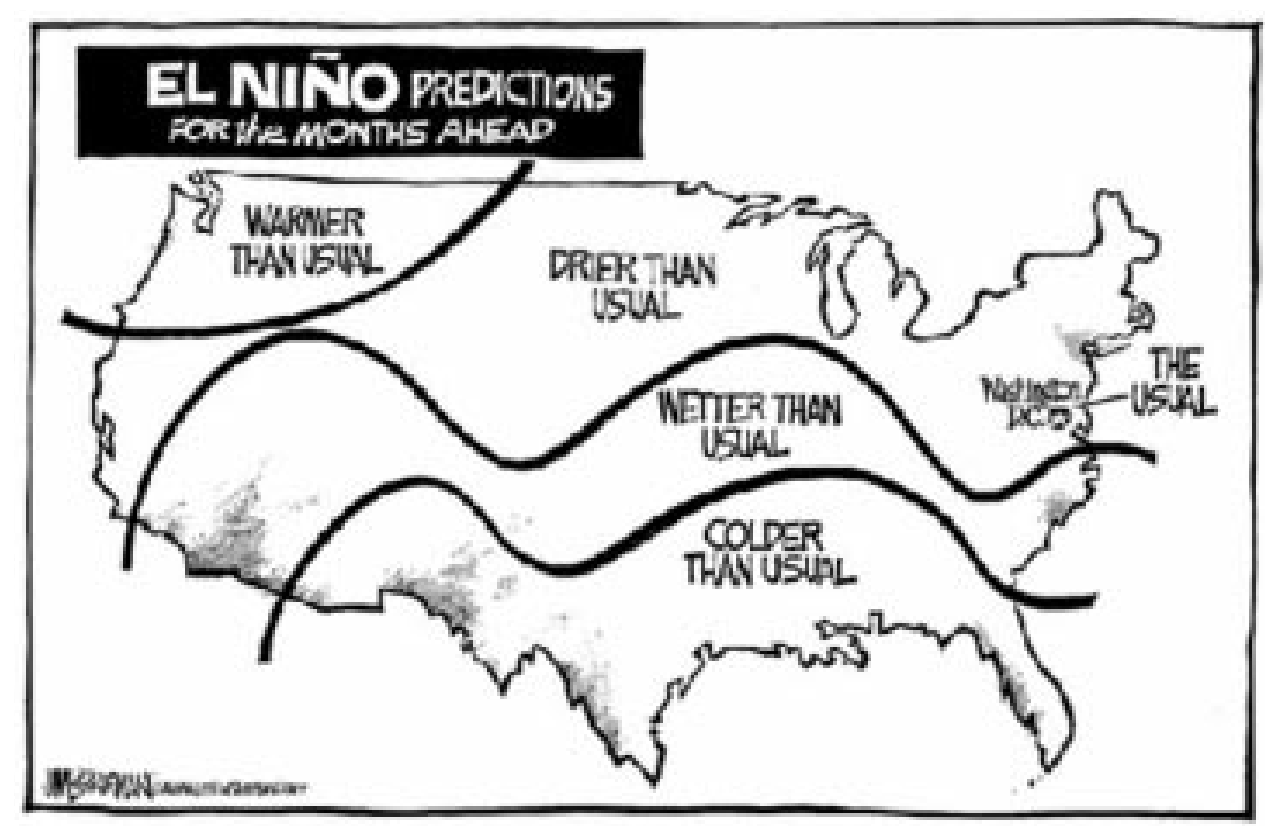

FIGURA 2. Viñeta cómica de Jim Borgman aparecida el 9 de abril de 1997 en el diario Cincinnati Enquirer, donde se hace referencia a los efectos de El Niño (más políticos que climáticos) sobre Estados Unidos.

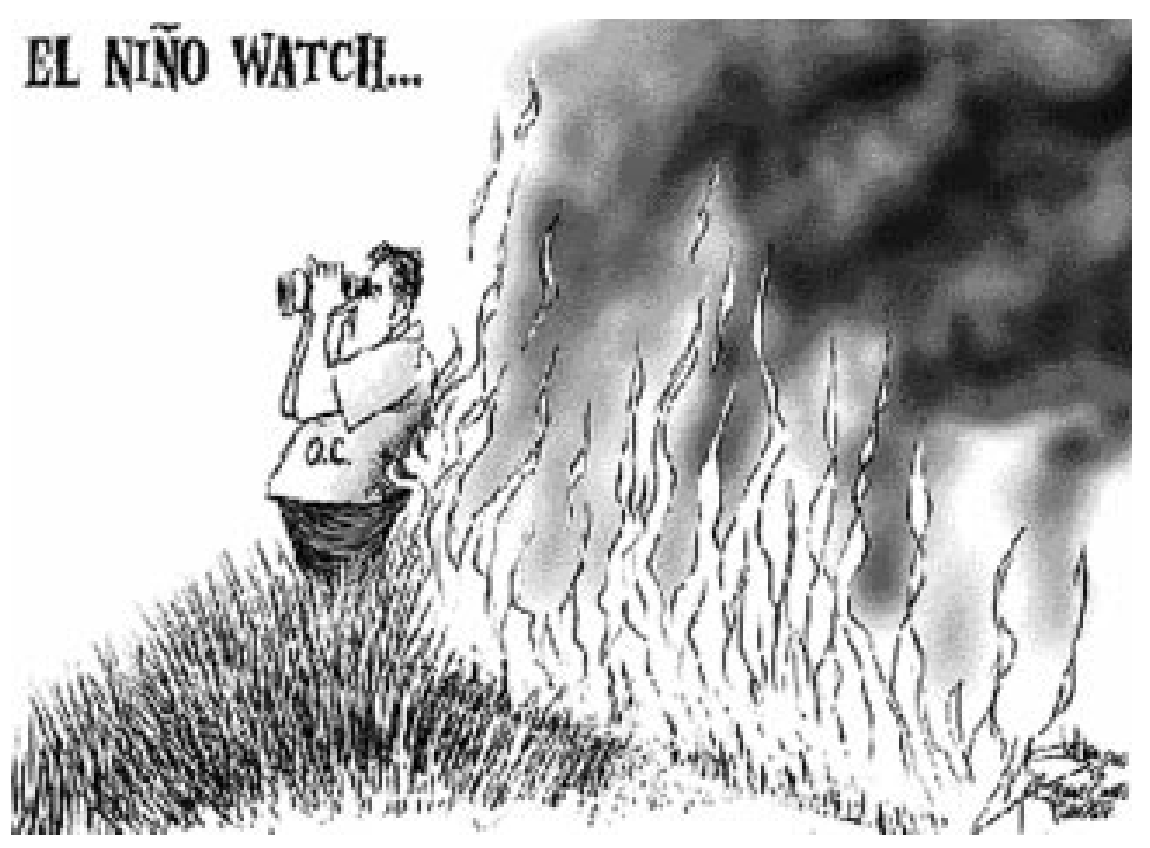

FIGURA 3. Viñeta cómica publicada el 15 de octubre de 1997 en el diario electrónico Liberty Online, publicación local del Orange County Register (Florida, Estados Unidos). 


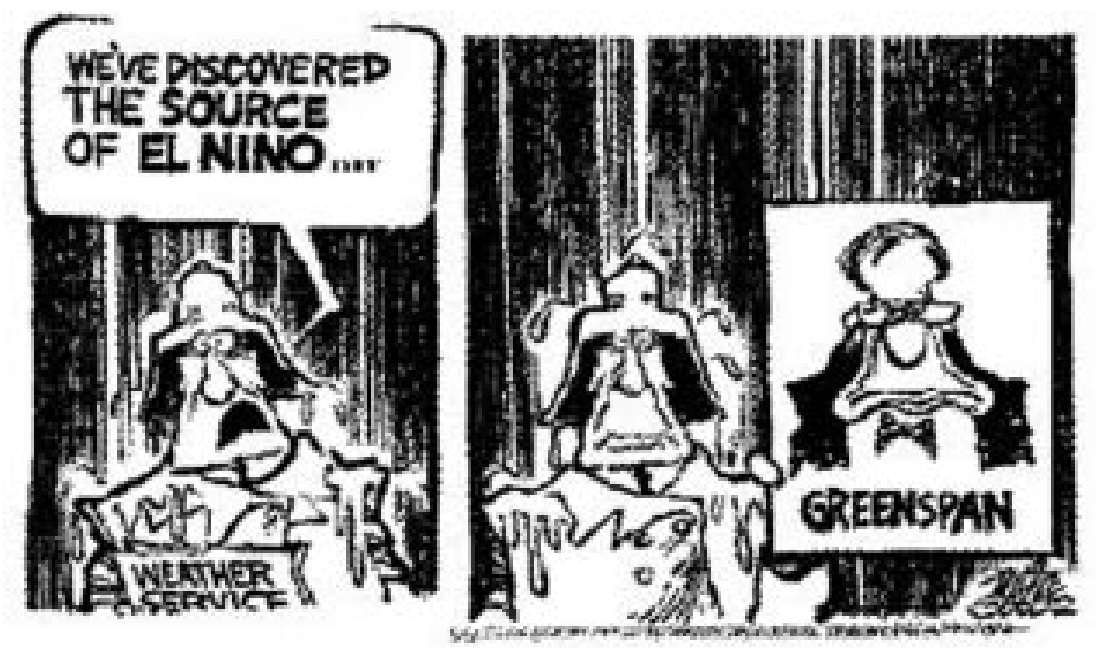

Figura 4. Viñeta cómica de Mike Greer, en la que el Servicio Meteorológico de Estados Unidos anuncia que ha encontrado el origen del fenómeno El Niño: el presidente de la Reserva Federal, Alan Greenspan.

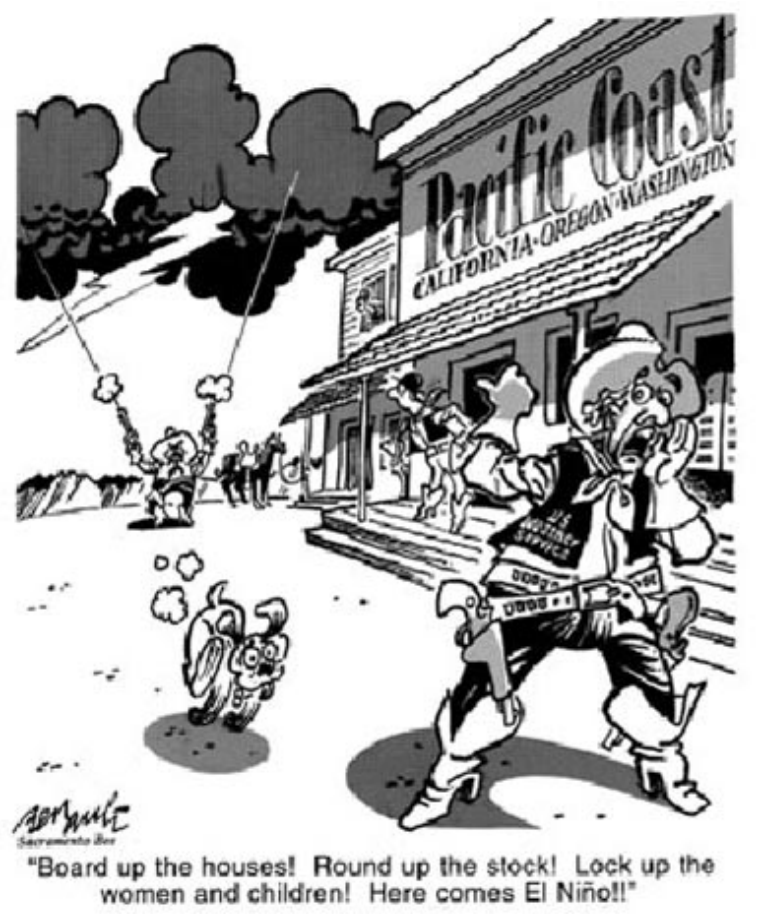

FiguRA 5. Viñeta cómica aparecida el 24 de agosto de 1997 en The Sacramento Bee, donde el Servicio Meteorológico de Estados Unidos (en forma de sheriff) avisa a la población de la llegada del fenómeno El Niño (en forma del conocido pistolero Billy the Kid). 
noticiarios de las principales cadenas de radio y televisión, documentales, CD-ROMs (REMEDIA, 1998) (ver figura $\mathrm{n}^{\circ}$ 1), e incluso viñetas humorísticas. En una búsqueda no exhaustiva a través de Internet, se han podido localizar siete historietas cómicas aparecidas en periódicos (Las Vegas Sun, Orange County Register, The Sacramento Bee, y otros), que relacionan El Niño con temas de actualidad política, como los problemas judiciales del presidente de Estados Unidos, Bill Clinton, o los constantes avisos de malos augurios para la bolsa lanzados por el presidente de la Reserva Federal, Alan Greenspan (ver figuras $n^{\circ} 2$ a 5).

Fuera de Estados Unidos también se han ofrecido informaciones jocosas, como en Radio Cuatro de la BBC británica, donde el 4 de marzo de 1998 se entrevistó a Al Nino, un piloto naval retirado de Nipomo (California), cuyo nombre presenta una gran similitud fonética con la pronunciación del término El Niño en inglés americano. La noticia señala que Al Nino, cuyo nombre aparece en la guía telefónica, recibió múltiples llamadas culpándole de las más diversas catástrofes.

El proceso de socialización del fenómeno El Niño creció progresivamente por sus efectos climáticos sobre diferentes zonas de Estados Unidos (tormentas, inundaciones y desprendimientos de tierras en California, tornados en Florida, temperaturas anómalamente suaves en el nordeste del país, etc.), de tal forma que casi toda la población se veía afectada en mayor o menor medida por el calentamiento del Pacífico ecuatorial. El interés informativo fue de tal magnitud ante el evento de 1997-98, que en el ránking de los diez acontecimientos informativos más destacados en el mundo durante el año 1997 elaborado por la CNN (1997), el Niño alcanzó el octavo lugar.

Sin embargo, la avalancha informativa provocó la aparición de noticias absurdas que desvirtúan la verdadera dinámica del fenómeno y producen falsas impresiones sobre El Niño entre la población, que puede llegar a considerarlo el causante de todos los males del momento. Las mayores exageraciones detectadas fueron las siguientes noticias:

- 20 de febrero de 1998, agencia Reuters: «Muerte de pollos atribuida a El Niño». La información culpa a El Niño de una ola de calor que provocó el fallecimiento de 20.000 pollos en diversas granjas del norte de Honduras.

- 27 de febrero de 1998, agencia Reuters: «El Niño visto como causa de gripe, plaga y revolución». El texto de la noticia es el siguiente: "Una conferencia de científicos australianos sobre El Niño ha señalado que el evento climático contribuyó a la Revolución Francesa, a la Peste Negra de finales de 1340, a intensos brotes epidémicos, y que podría ser la causa principal de la gripe. Richard Grove, coordinador de la conferencia «El Niño: Historia y Crisis» en la Universidad Nacional de Australia, dijo que los principales acontecimientos políticos y epidémicos de los últimos 5.000 años han estado relacionados con El Niño. [...] "Las condiciones sociales para la Revolución Francesa estaban presentes, pero si no hubiera sido por El Niño que aconteció en 1787-88, no habría habido Revolución".

- 12 de marzo de 1998, agencia Associated Press y CNN: «El Niño arrasa: la capital de la alcachofa cancela festival». La noticia precisa que "El Festival anual de la Alcachofa de Castroville (California) ha sido cancelado porque las lluvias de El Niño han arruinado la mayoría de la cosecha [...] ...las lluvias torrenciales han dejado al condado de Monterrey bajo el agua y han destruido el $40 \%$ de la cosecha de la región, valorada en 40 millones de dólares. Así que el festival, con una tradición de 39 años, será retrasado hasta mayo de 1999". Sin ser una afirmación falsa, el titular es claramente exagerado. 
Otro de los ámbitos en los que El Niño ha recibido una gran atención ha sido el económico, pero no por las repercusiones en la pérdida de cosechas o destrucción de infraestructuras, sino por las posibilidades de negocio para ciertos sectores. A continuación se muestran algunos ejemplos:

- 20/09/1997, San Francisco Chronicle: «Los reparadores de cubiertas abrumados de llamadas. La amenaza de El Niño impulsa un torrente de negocio». El reportaje muestra dos actividades económicas beneficiadas por la predicción de fuertes tormentas en California: contratistas de reparación de tejados, y pólizas de seguro con cobertura para inundaciones. En el texto del artículo se lee "Los contratistas de cubiertas dicen estar saturados de llamadas de propietarios en pánico reaccionando a las predicciones de feroces tormentas para este otoño e invierno".

- 19 de octubre de 1997, USA Today: «El Niño puede ayudar a algunas zonas de vacaciones". El artículo repasa los efectos climáticos de El Niño en zonas turísticas de todo el mundo, señalando algunas regiones favorecidas como el Caribe (menor número de huracanes) o el norte de Estados Unidos (temperaturas invernales más cálidas), y zonas perjudicadas como la Polinesia (intensa actividad tormentosa), o Indonesia (sequía, incendios y extensas nubes de humo).

- 08/12/1997, nota de prensa de Goodyear: «Goodyear engaña a El Niño». Esta compañía de neumáticos promocionó su producto con una nota de prensa, en la que recomendaban el uso de gomas Goodyear como ayuda ante el mal tiempo esperado en el sur de California y en el sudeste de Estados Unidos por efecto de El Niño.

- 09/12/1997, nota de prensa de la New England OverShoe Company: «Preparándose para El Niño: de la ropa de lluvia a kits de supervivencia». Esta compañía promociona unas botas resistentes a todo tipo de tiempo adverso, y especificamente ante las fuertes tormentas generadas por El Niño.

Un último grupo de noticias curiosas son las relacionadas con la actividad deportiva, en especial con el surf y el rafting. Sobre el primer deporte se contabilizaron diversas noticias, que coincidían en señalar que el Niño había generado grandes expectativas de oleaje en las playas de California, gracias a la constante entrada de tormentas procedentes del Pacífico.

- 13 de octubre de 1997, $C N N$ : «El Niño puede significar que el surf esté muy alto». En la noticia se destacan las grandes olas de El Niño de 1982-83, de hasta 10 metros, que causaron sensación entre los surfistas del momento.

- 15 de marzo de 1998, CNN: «El Niño impulsa olas peligrosas». Se destaca que la compañía K-2 Inc. organizó un concurso, ofreciendo un premio de 50.000 dólares para el surfista que cabalgara en la ola más grande generada por El Niño, y de 5.000 dólares para el fotógrafo que capturase el momento del récord.

- 30 de marzo de 1998, Time: «El invierno de las olas gigantes». El reportaje describe el resultado del concurso, cuyo ganador cabalgó una ola de más de 15 metros, y la opinión unánime de los surfistas: “¿Espero que El Niño regrese de nuevo el año próximo!".

- 13 de abril de 1998, CNN: «Guías fluviales se preparan para raftear El Niño». En la noticia se señala que El Niño creó condiciones inmejorables para el rafting en los ríos californianos, tanto por las abundantes lluvias como por el deshielo de la precipitación nival acumulada en el invierno. 
Del conjunto de noticias mostradas en este apartado, se deduce que El Niño ha sido utilizado para todo tipo de aplicaciones: como motivo de sátira, como excusa para justificar cualquier problema, elemento clave para la venta de productos, etc. Sin embargo, el lector no debe quedarse con una imagen poco rigurosa en los medios de comunicación estadounidenses, puesto que las noticias donde El Niño ha sido tratado con rigurosidad son infinitamente superiores a las aquí presentadas.

\section{Conclusiones}

El tratamiento informativo de cualquier temática de carácter científico suele producir reacciones negativas entre los investigadores, que perciben un lenguaje impreciso y múltiples exageraciones o deformaciones de la información original. Por lo que respecta a los periodistas, éstos son en ocasiones reacios a delegar las explicaciones en los científicos, por el temor de que la noticia no llegue al lector, oyente o espectador, por la excesiva complejidad de los plateamientos utilizados, el uso de tecnicismos y la falta de costumbre en la comunicación ciencia-sociedad.

El fenómeno El Niño de 1997-98, como se ha podido ver en este artículo, ha sido objeto de atención en todos los medios de comunicación, con el agravante de ser un evento natural desconocido por casi todos y que, antes de formar parte de la noticia, requería una breve presentación científica. La valoración general del tratamiento informativo de El Niño en España es bastante positiva, aunque hayan proliferado algunos equívocos graves (comunes a casi todos los medios de comunicación), como el de relacionar el fenómeno con el cambio climático y afirmar que El Niño se está agravando por efecto de la actividad humana.

Los ejemplos mostrados para Estados Unidos, exceptuando los tres casos de noticias donde El Niño adquiere el papel de chivo expiatorio, culpabilizándolo de acontecimientos inverosímiles, no son negativos de por sí, sino una muestra de la plena incorporación del fenómeno al lenguaje cotidiano.

Si en sucesivos eventos El Niño este proceso de total socialización observado en Estados Unidos se repite en España, debemos advertir sobre las distorsiones y exageraciones a que puede ser sometido el fenómeno. Actualmente, ya corremos el peligro de saturar a la población con múltiples conceptos de carácter medioambiental, de tal forma que el cambio climático, El Niño, el agujero en la capa de ozono, la polución atmosférica y otros términos, empiezan a confundirse entre sí peligrosamente.

Como siempre, la solución es difícil, y los consejos que podemos ofrecer desde un foro científico como este, son los que ya se han escuchado en anteriores ocasiones: el periodista debe extremar la precaución en el planteamiento de temas de carácter climátológico, que es el que nos ocupa, el científico debe hacerse accesible a los medios de comunicación y a la sociedad, simplificando su lenguaje, y ambos profesionales deben colaborar siempre que sea posible, de tal forma que el periodista acuda al científico cuando se le plantee alguna duda, y que el investigador consulte al reportero cómo hacer más accesibles sus explicaciones.

\section{Bibliografía}

Además de las referencias presentadas en este apartado, el lector encontrará noticias y artículos de prensa en los diarios y revistas (Abc, Avui, El País, El País Semanal, El Periódico de Catalunya, La Vanguardia) especificados a lo largo del artículo. 
CANBY, T.Y. (1984): «El Niño’s Ill Wind». National Geographic (edición EE.UU.), vol. $165, n^{\circ} 2$, pp. 144-183.

CAVIEDES, C.N. (1984): «Geography and the lessons from El Niño». The Professional Geographer, 36, pp. 428-436.

CNN (1997): «El Niño wreaks worldwide weather havoc». In: 1997 Year in Review. World, The 10 Top Stories of '97. Cable News Network. Atlanta. Documento en Internet: http://cnn.com/SPECIALS/1997/year.ender/world/08.el.nino.html.

DER SPIEGEL (1997): «El Niño, Wetter-Katastrophen werden vorhersehbar». Der Spiegel (Alemania), 13/10/1997, pp. 296-307.

ERCILLA (1997): «Un verano asombroso. Caos climático por El Niño». Ercilla (Chile), 17/11/1997, pp. 42-45.

LA VANGUARDIA (1999): La aventura de la ciencia. Enciclopedia multimedia. La Vanguardia. Barcelona. CD-ROM (vol. 3).

MARCOS, O. (1999): «Evolución en el estudio del fenómeno ENSO (El Niño - Oscilación del Sur): de anomalía 'local' a la predicción de variaciones climáticas globales». Investigaciones Geográficas (Alicante), nº 21, pp. 5-20.

MICROSOFT (1996): Microsoft Encarta 97 Encyclopedia. The American Heritage Concise Dictionary. ( $3^{\mathrm{a}}$ ed.). Microsoft. CD-ROM.

MASSONS, J. y CAMPS, J. (1998): «Del fuego al agua: El niño y La Niña». Mundo Científico, $\mathrm{n}^{\circ} 187$, pp. 59-65.

REMEDIA (1998): The El Niño CD-ROM. EarthGuides Series (Volume 1). Poway - California. REMedia Inc.

SAN FRANCISCO EXAMINER (1997): «An ill wind blown in again». San Francisco Examiner (Estados Unidos), 14/09/1997. Internet: http://www.sfgate.com/.

SUPLEE, C. (1999): «El Niño / La Niña. El ciclo vicioso de la naturaleza». National Geographic (edición española), vol. 4, n $\mathrm{n}^{\mathrm{3}}$ 3, pp. 74-97.

TIME (1997): «Is it El Nino of the century? Time (Estados Unidos), vol. 150, $\mathrm{n}^{\mathrm{o}} 7$, 18/08/1997. Internet, http://pathfinder.com/time/magazine/.

TIME (1998): «The fury of El Nino». Time (Estados Unidos), 16/02/1998, vol. 151, n 6. Internet: http://pathfinder.com/time/magazine/.

TYDEN (1998): «Stopy El Nina?». Tyden (República Checa), 14/04/1998, pp. 76-81. Internet: http://www.tyden.cz.

USA TODAY (1996): «Look back at 1994-95 El Niño». USA Today, 28 de marzo de 1996, edición electrónica en Internet (http://www.usatoday.com/weather/wnino2.htm).

U.S. NEWS (1997): «The power of El Niño». U.S. News (Estados Unidos), 6/10/1997. Internet: http://www.usnews.com/usnews/issue/971006/.

VIALARD, J. y QUENO, B. (1998): «El Niño. La corriente loca que altera el clima». Ciencia \& Vida, $\mathrm{n}^{\mathrm{o}} 3$, pp. 61-68.

VEJA (1997a): «Menino travesso». Veja (Brasil), 27/08/1997, pp. 42-45. Internet: http://www2.uol.com.br/veja/270897/p 042.html.

VEJA (1997b): «Foto do monstro». Veja (Brasil), 24/09/1997, p. 56. Internet, http://www2.uol.com.br/veja/240997/p 056.html.

VEJA (1997c): «A fúria natural». Veja (Brasil), 08/10/1997, pp. 102-107. Internet, http://www2.uol.com.br/veja/081097/p 102.html. 